Yet the theoretical impact of his work has been a game changer.

\section{Biographical Sketch}

Endre Szemerédi was born in Budapest, Hungary, in 1940. He received his Ph.D. from Moscow State University in 1970 under the direction of Israel M. Gelfand. He has held visiting positions at Stanford University (1974), McGill University (1980), the University of South Carolina (1981-1983), and the University of Chicago (1985-1986). He was the Fairchild Distinguished Scholar at the California Institute of Technology in 1987-1988. He is also a recipient of the Aisenstadt Chair at the Centre de Recherches Mathématiques, University of Montreal. In 2008 he was the Eisenbud Professor at the Mathematical Sciences Research Institute, Berkeley. He has received many awards and honors for his contributions to mathematics and computer science. In 2008 he was awarded the Leroy P. Steele Prize for Seminal Contribution to Research by the AMS. In the same year he received the Rolf Schock Prize in Mathematics from the Royal Swedish Academy of Sciences. Other prizes include the Grünwald Prize (1967, 1968), the Rényi Prize (1973), the Pólya Prize for Achievement in Applied Mathematics of the Society for Industrial and Applied Mathematics (SIAM) (1975), and the Prize of the Hungarian Academy of Sciences (1979). He has been a member of the Hungarian Academy of Sciences since 1987 and of the U.S. National Academy of Sciences since 2010 .

\section{About the Prize}

The Niels Henrik Abel Memorial Fund was established in 2002 to award the Abel Prize for outstanding scientific work in the field of mathematics. The prize is awarded by the Norwegian Academy of Science and Letters, and the choice of Abel Laureate is based on the recommendation of the Abel Committee, which consists of five internationally recognized mathematicians.

Previous recipients of the Abel Prize are: JeanPierre Serre (2003), Michael Atiyah and I. M. Singer (2004), Peter Lax (2005), Lennart Carleson (2006), S. R. S. Varadhan (2007), John G. Thompson and Jacques Tits (2008), Mikhail L. Gromov (2009), John Tate (2010), and John Milnor (2011).

- From announcements of the Norwegian Academy of Science and Letters

\title{
Pearl Receives 2011 Turing Award
}

The Association for Computing Machinery (ACM) has announced that JUDEA PEARL of the University of California, Los Angeles, has been named the recipient of the 2011 ACM A. M. Turing Award "for fundamental contributions to artificial intelligence through the development of a calculus for probabilistic and causal reasoning." The award carries a cash prize of US\$250,000 and will be presented to Pearl at the annual ACM Awards Banquet on June 16, 2012, in San Francisco, California.

\section{Citation}

Judea Pearl's work has transformed artificial intelligence (AI) by creating a representational and computational foundation for the processing of information under uncertainty. Pearl's work went beyond both the logic-based theoretical orientation of AI and its rule-based technology for expert systems. He identified uncertainty as a core problem faced by intelligent systems and developed an algorithmic interpretation of probability theory as

DOI: http://dx.doi.org/10.1090/noti854 an effective foundation for the representation and acquisition of knowledge.

Focusing on conditional independence as an organizing principle for capturing structural aspects of probability distributions, Pearl showed how graph theory can be used to characterize conditional independence and invented message-passing algorithms that exploit graphical structure to perform probabilistic reasoning effectively. This breakthrough has had major impact on a wide variety of fields where the restriction to simplified models had severely limited the scope of probabilistic methods; examples include natural language process-

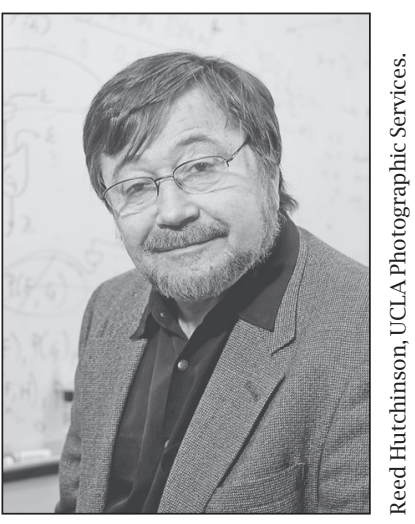
Judea Pearl

ing, speech processing, computer vision, robotics, computational biology, and error-control coding.

Equally significant is Pearl's work on causal reasoning, where he developed a graph-based calculus of interventions that makes it possible to derive 
EIH

Eidgenössische Technische Hochschule Zürich

Swiss Federal Institute of Technology Zurich

\section{Professor of Applied} Mathematics

The Department of Mathematics (www.math.ethz.ch) at ETH Zurich invites applications for a position in Applied Mathematics at the Full or Associate Professor level. The vacant position is within the Seminar for Applied Mathematics, SAM (www.sam.math.ethz.ch).

The successful candidate's mathematical results should have received wide international recognition. His or her results should be landmark contributions to mathematical modelling and/or efficient numerical simulation in engineering and the sciences. A strong algorithmic and computational component in his/her mathematical research is expected. The candidate should have demonstrated proficiency in conducting pioneering projects in applied mathematics. Together with other members of the Department, the new professor will be responsible for teaching undergraduate courses (German or English) and graduate courses (English) for students in Applied Mathematics and Computational Science and Engineering (CSE).

Please apply online at www.facultyaffairs.ethz.ch. Your application should include your curriculum vitae and a list of publications. The letter of application should be addressed to the President of ETH Zurich, Prof. Dr. Ralph Eichler. The closing date for applications is 31 July 2012. ETH Zurich is an equal opportunity and affirmative action employer. In order to increase the number of women in leading academic positions, we specifically encourage women to apply. ETH Zurich is further responsive to the needs of dual career couples and qualifies as a family friendly employer. causal knowledge from the combined effects of actions and observations. This work has been transformative within AI and computer science and has had major impact on allied disciplines of economics, philosophy, psychology, sociology, and statistics.

\section{Biographical Sketch}

Judea Pearl is a professor of computer science at the University of California, Los Angeles, where he was director of the Cognitive Systems Laboratory. Before joining UCLA in 1970, he was at RCA Research Laboratories working on superconductive parametric and storage devices. Previously, he was engaged in advanced memory systems at Electronic Memories, Inc. Pearl is a graduate of the Technion, the Israel Institute of Technology, with a B.S. degree in electrical engineering. In 1965 he received a master's degree in physics from Rutgers University and in the same year was awarded a Ph.D. degree in electrical engineering from the Polytechnic Institute of Brooklyn.

Among his many awards, Pearl is the recipient of the 2012 Harvey Prize in Science and Technology from the Technion and the 2008 Benjamin Franklin Medal in Computers and Cognitive Science from the Franklin Institute. He was presented with the 2003 Allen Newell Award from ACM and the AAAI (Association for the Advancement of Artificial Intelligence). His groundbreaking book on causality, Causality: Models, Reasoning, and Inference, won the 2001 Lakatos Award from the London School of Economics and Political Science "for an outstanding significant contribution to the philosophy of science."

Pearl is a member of the National Academy of Engineering and a Fellow of AAAI and the Institute for Electrical and Electronic Engineers (IEEE). He is president of the Daniel Pearl Foundation, named after his son.

\section{About the Award}

The A. M. Turing Award was named for Alan M. Turing, the British mathematician who articulated the mathematical foundation and limits of computing and who was a key contributor to the Allied cryptanalysis of the German Enigma cipher during World War II. Since its inception in 1966, the Turing Award has honored the computer scientists and engineers who created the systems and underlying theoretical foundations that have propelled the information technology industry.

The award is given to an individual selected for contributions of a technical nature made to the computing community. The contributions should be of lasting and major technical importance to the computer field. Financial support for the Turing Award is provided by the Intel Corporation and Google Inc.

- ACM announcement 